\title{
Interaction between Warfarin and Proton Pump Inhibitors
}

\author{
Takeshi Shirayama, Hirokazu Shiraishi, Akira Kuroyanagi, Tetsuro Hamaoka, \\ Mikimasa Imai, Akimitsu Kojima \\ Department of Cardiology, Kyoto Prefectural University of Medicine, Kyoto, Japan \\ Email: sirayama@koto.kpu-m.ac.jp
}

Received 5 May 2014; revised 4 June 2014; accepted 3 July 2014

Copyright (C) 2014 by authors and Scientific Research Publishing Inc.

This work is licensed under the Creative Commons Attribution International License (CC BY). http://creativecommons.org/licenses/by/4.0/

(c) (i) Open Access

\begin{abstract}
Background: Interaction between proton pump inhibitors (PPI) and warfarin is controversial. Previous clinical studies have only a short follow-up period. Methods and Results: All patients (n = 716) for whom warfarin was prescribed from November 1, 2010 to October 30, 2011 were extracted from electronic health records. In retrospective analysis for 1 year, PPI were prescribed to 404 patients. Among them, 108 patients were taking warfarin for more than 6 weeks before and after PPI. The profile of these patients was analyzed: 63 patients took lansoprazole; 15 patients took omeprazole; 30 patients took rabeprazole. No statistical difference was observed among 3 groups in age, body weight, concomitant use of other drugs, and comorbidity. Warfarin dose and INR did not change after PPI. Multivariate stepwise logistic regression analysis revealed that upper quartile of increment of INR was associated with the presence of atrial fibrillation (OR 3.77, 95\% CI 1.16 - 12.27). The patients who had warfarin for shorter periods before PPI, or those who had PPI first $(n=141)$ had similar dose of warfarin and INR. In all patients analyzed $(n=404)$, including patients whose follow-up periods were shorter than 6 weeks $(n=155)$, a patient had cerebral bleeding, and 2 patients had cerebral infarction. Conclusions: Unfavorable interaction between warfarin and PPI was negligible in clinical use. Relatively higher INR was achieved after PPI in the presence of atrial fibrillation.
\end{abstract}

\section{Keywords}

Warfarin, Proton Pump Inhibitor, Interaction

\section{Introduction}

Atrial fibrillation is a major cause of systemic embolism, which often results in cerebral infarction with severe 
sequelae [1]. Warfarin is used to prevent cardiogenic embolism for the patients at risk [1]-[3]. The other major indications are deep vein thrombosis and mechanical valve replacement, which large numbers of the patients suffer from. Proton pump inhibitors (PPI) are also widely used because the number of patients with esophageal regurgitation is increasing and the prevention of aspirin-ulcer has been more and more important [4]. Cardiologists thus often use these drugs concomitantly [5].

Drug interaction of warfarin and PPI is a very important issue, because warfarin inhibits competitively the metabolic enzymes of PPI for their degradation [6]. For example, R-warfarin inhibits CYP2C19 [7] [8], which metabolizes most of lansoprazole [9] and omeprazole [10]. It also metabolizes a part of rabeprazole [6]. However, it is not known how this pharmacological difference among PPI is relevant to the clinical practice. In fact, there are few clinical studies on this matter [11]-[14]. Some studies pointed out that PPI increased the anti-coagulation effect of warfarin [11] [14], i.e., INR was increased, but their observation periods were too short (1 to 2 weeks) to draw a definite conclusion. The effect of warfarin is known to fluctuate during a long-term follow-up where adverse effects could be seen [15].

We studied the interaction of warfarin and 3 PPI by the retrospective observation for relatively long-term.

\section{Methods}

\subsection{Patients}

All patients who received warfarin and one of PPI from November 1, 2010 to October 30, 2011 were extracted from the electrical health records in our institution $(n=716)$.

\subsection{Analysis}

Main analysis was performed using the data of the patients who received warfarin more than 6 weeks before the initial administration of a PPI. Information on demographic data, underlying disease, NSAIDS and/or antiplatelet drug use, time course of warfarin dose and prothrombin time (INR), complications were collected, dividing into 3 drug groups of PPI (lansoprazole, omeprazole, rabeprazole). The other PPI were not included because they were not available in Japan during the study period. A linear logistic regression analysis was done to find factors that affected the warfarin effects.

A supplementary analysis was also done for the patients who received warfarin first but equal to or less than 6 weeks prior to PPI administration, and the patients who received PPI first $(n=141)$. Mean warfarin dose and INR was calculated for these patients. Because warfarin dose could be adjusted along with the time course in the presence of PPI, they were excluded from a logistic regression analysis for the factors affecting warfarin dose and INR.

Only side effects were studied for the patients who received warfarin and PPI for less than 6 weeks $(n=155)$. The patients who did not receive warfarin and PPI simultaneously were excluded from the analysis $(n=312)$.

\subsection{Statistics}

Data are shown as mean \pm SD in the text, and median (the first and the third quartile) in some figures. Statistical analysis was performed using ANOVA for the difference of demographic data among 3 groups, Friedman test for the time-course of warfarin dose and INR within the same PPI group, Kruskal-Wallis test for the comparison among 3 groups during the time course. Chi-square test was performed when appropriate. Univariate and multivariate logistic regression analysis was performed for the main analysis to find out the factors that affected an anti-thrombotic effect of warfarin. $\mathrm{P}<0.05$ was considered statistically significant. Confidential intervals were shown in the presentation of odds ratio. The protocol of this study was approved by the ethics committee of our institution.

\section{Results}

\subsection{Main Results}

The subjects of this analysis were the patients who had a sufficient period of warfarin dose adjustment (>6 weeks) prior to the introduction of PPI $(n=108)$. Table 1 shows age, sex, body weight, underlying diseases for the warfarin requirement, drug use that could affect the anti-coagulation in each PPI group. There was no dif- 
ference among 3 groups.

Time-course of prothrombin time (INR) before and after PPI introduction was shown in Figure 1. The follow-up period was at least 12 weeks for omeprazole, at least 16 weeks for lansoprazole and rabeprazole. Average INR before PPI administration were $1.89 \pm 0.50$ for lansoprazole group $(n=63), 1.80 \pm 0.68$ for omeprezole group $(n=15)$, and $1.78 \pm 0.48$ for rabeprazole group $(n=30)$, respectively (not statistically significant). PPI

Table 1. Comparison of patients' characteristics.

\begin{tabular}{ccccc}
\hline & Lansoprazole & Omeprazole & Rabeprazole & P value $^{*}$ \\
\hline Patient number & 63 & 15 & 30 & \\
Age (years old) & $71.2 \pm 11.3$ & $74.1 \pm 8.9$ & $71.0 \pm 12.1$ & 0.6420 \\
Body weight (kg) & $60.0 \pm 12.1$ & $63.5 \pm 15.4$ & $60.5 \pm 12.5$ & 0.6310 \\
Male [\%] & $40[63.5]$ & $10[66.7]$ & $24[80.0]$ & 0.2732 \\
Atrial fibrillation [\%] & $37[58.7]$ & $8[53.3]$ & $14[6.7]$ & 0.5474 \\
Ventricular tachycardia [\%] & $11[17.5]$ & $3[20.0]$ & $6[20.0]$ & 0.9454 \\
Valvular heart disease [\%] & $16[25.4]$ & $4[26.7]$ & $6[20.0]$ & 0.8237 \\
Cardiac valve replacement [\%] & $8[12.7]$ & $3[20.0]$ & $5[16.7]$ & 0.7317 \\
Cardiomyopathy [\%] & $7[11.1]$ & $0[0]$ & $2[6.7]$ & 0.3484 \\
Myocardial infarction [\%] & $17[27.0]$ & $5[33.3]$ & $6[20.0]$ & 0.6023 \\
Angina pectoris [\%] & $6[9.5]$ & $0[0]$ & $4[13.3]$ & 0.3450 \\
Amiodarone [\%] & $3[4.8]$ & $0[0]$ & $2[6.7]$ & 0.6027 \\
Antiplatelet [\%] & $11[17.5]$ & $2[13.3]$ & $7[23.3]$ & 0.6788 \\
Aspirin [\%] & $27[42.9]$ & $6[40.0]$ & $11[36.7]$ & 0.8494 \\
NSAIDS [\%] & $11[17.5]$ & $2[13.3]$ & $4[13.3]$ & 0.8449 \\
\hline
\end{tabular}

P-value*: Chi-square test or Kruskal-Wallis test.

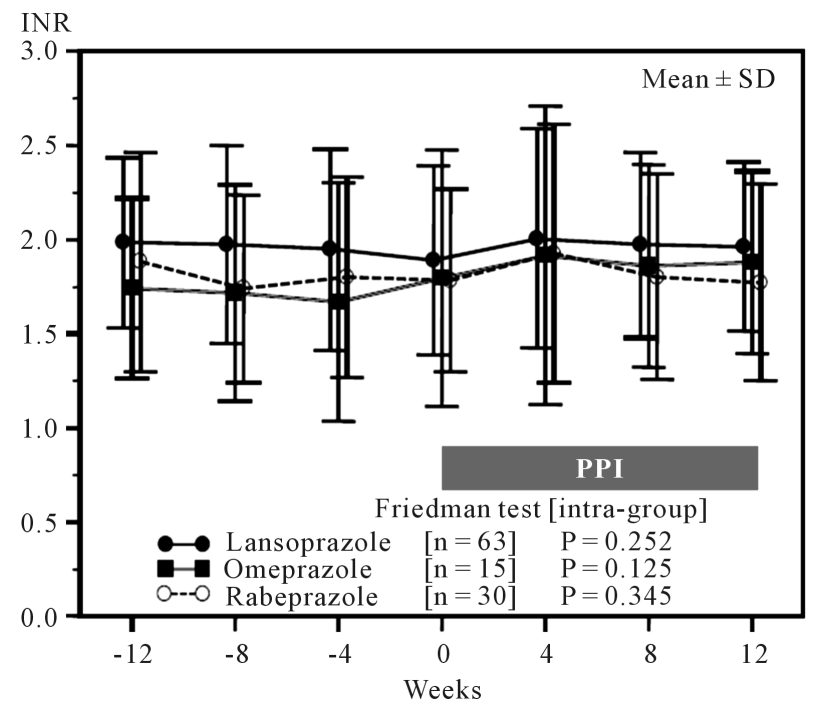

Figure 1. Changes in INR before and after PPI: The average INR were plotted against time-course of each PPI group. At the week 0 , PPI was introduced to the patient. No significant changes were observed in all groups. 
did not change INR during the treatment in 3 groups.

Dose adjustment of warfarin was shown in Figure 2. Average dose was $2.99 \pm 1.26 \mathrm{mg}$ for lansoprazole group, $2.95 \pm 0.81 \mathrm{mg}$ for omeprazole group, and $2.68 \pm 0.94 \mathrm{mg}$ for rabeprazole group. In average, there was no statististical difference in warfarin dose after PPI administration.

Visit-to-visit changes of INR after the introduction of PPI are shown in Figure 3. Standard deviation for the entire time-course was divided by mean INR in each patient, and the distribution of these data was plotted.

There was a tendency that the changes were greater in lansoprazole group, but the difference was less than $50 \%$. There was no statistical difference among 3 groups.

Maximal change of INR in each patient after the introduction of PPI was shown in Figure 4. Median change was within \pm 0.5 , and there was no statistical difference among 3 groups. Bar graphs demonstrate that INR swung to the smaller number more often than the larger number. INR of some patients, however, increased by more than 1. Patient-to-patient difference seemed rather greater in lansoprazole group. The percentage of the upper quartile ( $>75 \%)$ of maximal INR change $(>0.690)$ during the time course was compared in Figure 5, but the difference was not statistically significant among 3 drug groups.

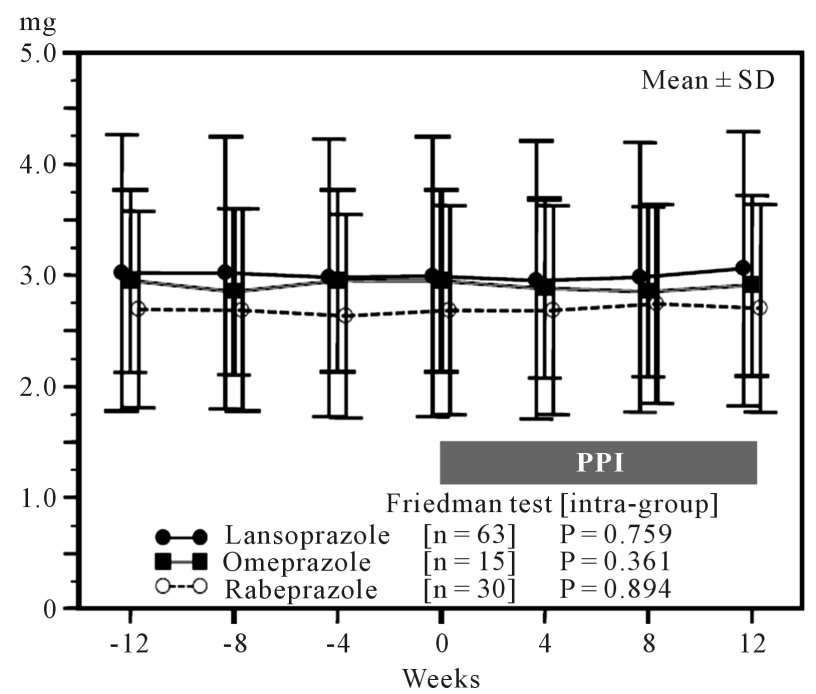

Figure 2. Changes in warfarin dose before and after PPI: The average doses of warfarin were plotted against time-course of each PPI group, as shown in Figure 1. Dose was not changed after PPI introduction.

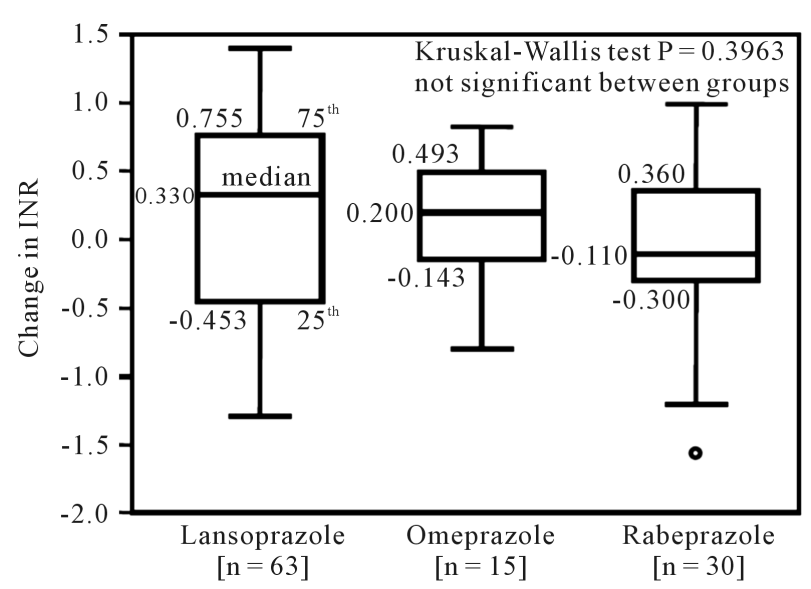

Figure 3. Visit-to-visit INR variability: The range of INR variability of each patient was evaluated as standard deviation over mean INR. Variability was not different in all groups. 


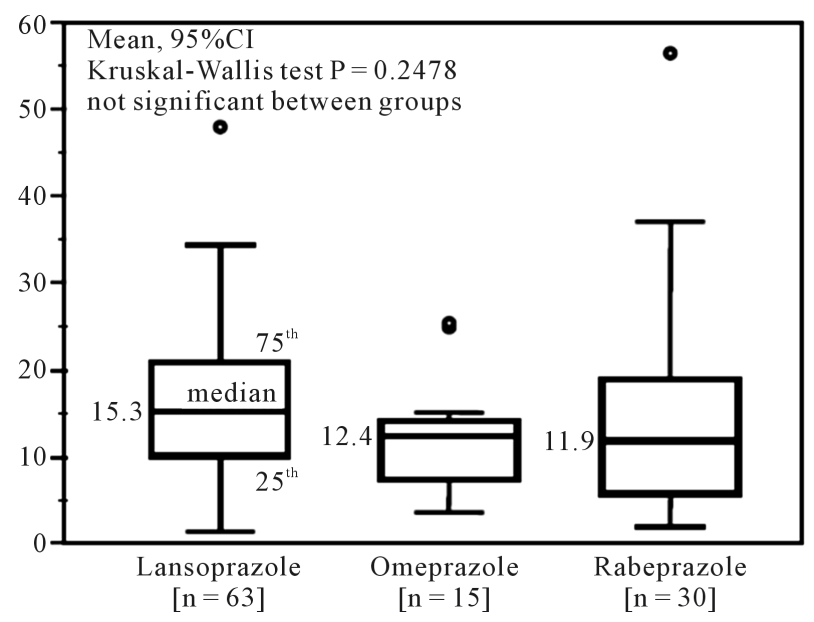

Figure 4. Maximum change of INR after PPI introduction: The maximum change of INR during follow-up period after PPI introduction was compared among each PPI group. There was no significant difference among 3 groups.

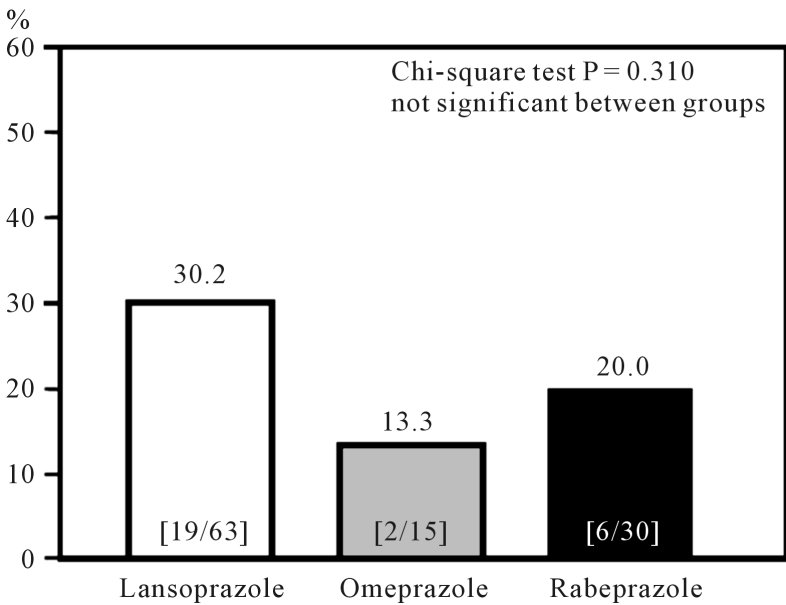

Figure 5. Percentage of the patients who was in the upper quartile of INR increase: Some patients showed relatively large increase of INR after the introduction of PPI. When the patients showed $>0.690$ increase of INR, they were in the upper-most quarter of all study population. The distribution of these patients in each PPI group is shown in the figure. INR of the patients in the labeprezole group tended to increase larger than INR in other groups, but it was not significant.

Univariate logistic regression analysis was performed to find the conditions that could affect the INR increase (Table 2). When the upper quartile increase of INR $(>0.690)$ is set to a dependent variable, the presence of atrial fibrillation had the odds ratio >3.0 (CI 1.16 - 12.27). The same result was obtained when a multivariate analysis was performed (Table 3).

\subsection{Supplementary Analysis}

Analysis was done for the patients for whom the add-on effects of PPI to warfarin could not be evaluated because of the short period ( $<6$ weeks) before PPI introduction or the presence of PPI administration before warfarin. Eighty (80) patients for lansoprazole, 22 patients for opmeprazole and 39 patients for rabeprazole were identified for this analysis. The average INR was $1.84 \pm 0.42$ for lansoprazole group, $1.80 \pm 0.36$ for omeprazole 
Table 2. Univariate logistic regression analysis.

\begin{tabular}{cccc}
\hline Variable & Odds ratio & $95 \%$ CI & P-value \\
\hline Age & 1.0078 & $0.9605-1.0575$ & 0.7511 \\
Gender [male vs female] & 1.0000 & $0.3225-3.1006$ & 0.9923 \\
Atrial fibrillation & 3.7692 & $1.1579-12.2702$ & 0.0227 \\
Ventricular tachycardia & 0.7187 & $0.1447-3.5689$ & 0.6863 \\
Valvular heart disease & 1.0000 & $0.3109-3.2163$ & 0.9943 \\
Cardiac valve replacement & 2.0125 & $0.5130-7.8951$ & 0.3159 \\
Cardiomyopathy & 1.8182 & $0.3883-8.5140$ & 0.4415 \\
Myocardial infarction & 1.3068 & $0.3100-5.5095$ & 0.7148 \\
Angina pectoris & 1.0250 & $0.9611-1.0931$ & 0.4522 \\
Amiodarone & 0.4808 & $0.0410-5.6413$ & 0.5599 \\
Antiplatelet & 0.3520 & $0.0620-1.9996$ & 0.2387 \\
Aspirin & 1.3750 & $0.4537-4.1676$ & 0.5735 \\
NSAIDS & 1.0000 & $0.2228-4.4889$ & 0.9921 \\
Lansoprazole vs other PPI & 1.1875 & $0.3760-3.7506$ & 0.7696 \\
\hline
\end{tabular}

Dependent variable $=$ upper quartile $\left(75^{\text {th }}\right)$ of INR increment $>0.690$.

Table 3. Multivarite logistic regression analysis.

\begin{tabular}{cccc}
\hline Variable & Odds ratio & $95 \%$ CI & P-value \\
\hline Age & 0.9961 & $0.9305-1.0662$ & 0.9095 \\
Gender [male vs female] & 1.0915 & $0.2306-5.1675$ & 0.9121 \\
Atrial fibrillation & 15.1362 & $1.5243-150.3010$ & 0.0202 \\
Ventricular tachycardia & 6.7423 & $0.2719-167.1845$ & 0.2440 \\
Valvular heart disease & 0.4054 & $0.0174-9.4225$ & 0.5738 \\
Cardiac valve replacement & 8.5921 & $0.2555-288.9540$ & 0.2305 \\
Cardiomyopathy & 3.7666 & $0.3353-42.3062$ & 0.2825 \\
Myocardial infarction & 7.4395 & $0.4903-112.8927$ & 0.1481 \\
Angina pectoris & 6.3843 & $0.3530-115.4705$ & 0.2095 \\
Amiodarone & 0.1674 & $0.0065-4.3037$ & 0.2806 \\
Antiplatelet & 0.4582 & $0.0526-3.9959$ & 0.4800 \\
Aspirin & 1.2026 & $0.2372-6.0981$ & 0.8237 \\
NSAIDS & 2.6302 & $0.2860-24.1870$ & 0.3929 \\
Lansoprazole vs other PPI & 1.4527 & $0.2936-7.1884$ & 0.6471 \\
\hline
\end{tabular}

Dependent variable $=$ upper quartile $\left(75^{\text {th }}\right)$ of INR increment $>0.690$.

group and $1.88 \pm 0.52$ for rabeprazole group, respectively. The average dose of warfarin was $2.57 \pm 1.35 \mathrm{mg}$ for lansoprazole group, $2.32 \pm 0.63 \mathrm{mg}$ for omeprazole group and $2.65 \pm 1.54 \mathrm{mg}$ for rabeprazole group, respectively. These numbers are not different from the patients of main analysis. There was no difference among 3 PPI groups. 


\subsection{Side Effects}

All ischemic and major bleeding events were investigated in all patients who received warfarin and PPI simultaneously $(\mathrm{n}=404)$. In addition to the patients for the analysis described above, 155 cases who received warfarin and PPI only for a short period ( $<6$ weeks) were included. There were 2 cases of cerebral infarction. Warfarin and omeprazole were started simultaneously for them. Age/INR/warfarin dose were 59 years old/1.48/1.5 mg and 70 years old/1.76/2.75 mg, respectively. A patient had cerebral bleeding after warfarin and lansoprazole were started simultaneously. Age/INR/warfarin dose were 80 years old/2.51/3 mg. The conditions of these patients were not severe and they were treated conservatively.

\section{Discussion}

Proton pump inhibitors (PPI) are metabolized by cytochrome P450. However, metabolic pathways for each PPI are slightly different. Lansoprazole and omeprazole are metabolized mainly by CYP2C19 and partly by CYP3A4 [8]-[10], whereas rabeprazole is metabolized partly by CYP2C19 but non-enzymatically in most part [6]. Because R-warfarin (active stereoisomer of warfarin) is metabolized by CYP2C19 among others [9] [10], concomitant use of warfarin and PPI, especially lansoprazole and omeprazole, could result in the competitive inhibition of metabolism and in turn the effect of the agents become intensified. In this scenario, clinically relevant concern is the increased anti-coagulation activity, i.e., the bleeding tendency. In our knowledge, there have been very few reports on this subject. The previous reports are either episodic case reports (presentation in congress) or observations for very short period ( $<2$ weeks) [8]-[14].

This study showed that the use of PPI on top of warfarin did not change anti-coagulation action of warfarin for more than 6 weeks. There was a tendency of slight increase of INR after the addition of PPI, but the difference was small. The fluctuation of anti-coagulation action was also small enough to require special attention in clinical situations. The difference among 3 PPIs were not discernible. These observations were the case when we looked at the patients who had PPI first. The average dose of warfarin and INR were not different from those of the patients who had warfarin first.

In some cases, the anti-coagulation effect of warfarin was fluctuated [15], although the maximum change was within acceptable range. Multivariate analysis revealed that the only factor that predicted the larger fluctuation was the presence of atrial fibrillation. The clinical reasoning of this observation was difficult to determine in our data. It is possible that the frequency of paroxysm, heart rate control, and change of anti-arrhythmic drugs could affect the status of heart failure, liver congestion and the metabolism of warfarin.

Cerebrovascular complications occurred in 3 cases among a total of 404 cases during 1 year period in this study. This is a relatively low percentage compared with the other studies [1] [2]. This result also warranted safety of the concomitant use of warfarin and PPI. Notwithstanding, it seems to be safe to say that clinicians should be careful when they start 2 drugs simultaneously as in 3 cases mentioned above.

Major limitation of this study is the fact that this is a retrospective observation. However, we included all patients for the analysis. If PPI had any discernible interactions with warfarin, it should be detected in this study. Indeed, INR was not changed after the introduction of PPI. Physicians could change warfarin dose, but it was not remarkable as shown in Figure 2. We have no control group during the entire observation period, but we can show a sufficient length of control periods as seen in Figure 1 and Figure 2. Many drugs (digoxin, cyclosporine, etc.) have interactions with warfarin, but such interactions were not surveyed in this study.

\section{Conclusion}

In conclusion, concomitant use of PPI and warfarin is safe and no special attention is required. No dose-adjustment of warfarin is necessary after the introduction of PPI.

\section{Disclosures}

No conflict of interest to be reported.

\section{References}

[1] Hart, R.G., Pearce, L.A. and Aguilar, M.I. (2007) Meta-Analysis: Antithrombotic Therapy to Prevent Stroke in Patients Who Have Nonvalvular Atrialfibrillation. Annals of Internal Medicine, 146, 857-867. 
http://dx.doi.org/10.7326/0003-4819-146-12-200706190-00007

[2] Ogawa, S., Koretsune, Y., Yasaka, M., Aizawa, Y., Atarashi, H., Inoue, H., et al. (2011) Antithrombotic Therapy in Atrial Fibrillation: Evaluation and Positioning of New Oral Anticoagulant Agents. Circulation Journal, 75, 1539-1547. http://dx.doi.org/10.1253/circj.CJ-11-0304

[3] Doufekias, E., Segal, A.Z. and Kizer, J.R. (2008) Cardiogenic and Aortogenic Brain Embolism. Journal of the American College of Cardiology, 51, 1049-1059. http://dx.doi.org/10.1016/j.jacc.2007.11.053

[4] Lanza, F.L., Chan, F.K.L. and Quigley, E.M.M. (The Practice Parameters Committee of the American College of Gastroenterology) (2009) Guidelines for Prevention of NSAID-Related Ulcer Complications. American Journal of Gastroenterology, 104, 728-738. http://dx.doi.org/10.1038/ajg.2009.115

[5] Hungin, A.P., Rubin, G.P. and O’Flanagan, H. (1999) Co-Prescription of $\mathrm{H}_{2}$ Receptor Blockers and Proton Pump Inhibitors with Warfarin in General Practice. Postgraduate Medical Journal, 75, 721-722.

[6] Li, X.Q., Andersson, T.B., Ahlström, M. and Weidolf, L. (2004) Comparison of Inhibitory Effects of the Proton Pump Inhibiting Drugs. Omeprazole, Edomeprazole, Lansoprazole, Pantoprazole and Rabereazole on Human Cytochrome P450 Activities. Drug Metabolism and Disposition, 32, 821-827. http://dx.doi.org/10.1124/dmd.32.8.821

[7] Zhou, Q. and Chan, E. (2003) Effect of Omeprazole on the Anticoagulant Activity and the Pharmacokinetics of Warfarin Enantiomers in Rats. European Journal of Pharmaceutical Sciences, 20, 439-449. http://dx.doi.org/10.1016/j.ejps.2003.09.007

[8] Zhou, Q., Zhou, S. and Chan, E. (2005) Effect of Omeprazole on the Hydroxylation of Warfarin Enantiomers in Human: In-Vitro Studies with Liver Microsomes and cDNA-Expressed Cytochrome P450 Isozymes. Current Drug Metabolism, 6, 399-411. http://dx.doi.org/10.2174/138920005774330620

[9] Hata, M., Hayasaka, M., Sezai, A., Niino, T., Yoda, M., Unosawa, S., et al. (2008) Proton Pump Inhibitors May Increase the Risk of Delayed Bleeding Complications after Open Heart Surgery If Used Concomitantly with Warfarin. The Thoracic and Cardiovascular Surgery, 56, 274-277. http://dx.doi.org/10.1055/s-2008-1038378

[10] Uno, T., Sugimoto, K., Sugawara, K. and Tateishi, T. (2008) The Role of Cytochrome P2C19 in R-Warfarin Pharmacokinetics and Its Interaction with Omeprazole. Therapeutic Drug Monitoring, 30, 276-281. http://dx.doi.org/10.1097/FTD.0b013e31816e2d8e

[11] Teichert, M., van Noord, C., Uitterlinden, A.G., Hofman, A., Buhre, P.N., De Smet, P.A., et al. (2011) Proton Pump Inhibitors and the Risk of Overanticoagulation during Acenocoumarol Maintenance Treatment. British Journal of Haematology, 153, 379-385. http://dx.doi.org/10.1111/j.1365-2141.2011.08633.x

[12] Sutfin, T., Balmer, K., Boström, H., Eriksson, S., Höglund, P. and Paulsen, O. (1989) Stereoselective Interaction of Omeprazole with Warfarin in Healthy Men. Therapeutic Drug Monitoring, 11, 176-184. http://dx.doi.org/10.1097/00007691-198903000-00010

[13] Unge, P., Svedberg, L.E., Nordgren, A., Blom, H., Andersson, T., Lagerström, P.O., et al. (1992) A Study of the Interaction of Omeprazole and Warfarin in Anticoagulated Patients. British Journal of Clinical Pharmacology, 34, 509512. http://dx.doi.org/10.1111/j.1365-2125.1992.tb05656.x

[14] Moffett, B.S., Ung, M. and Bomgaars, L. (2012) Risk Factors for Elevated INR Values during Warfarin Therapy in Hospitalized Pediatric Patients. Pediatric Blood Cancer, 58, 941-944. http://dx.doi.org/10.1002/pbc.23308

[15] Flockhart, D.A., O’Kane, D., Williams, M.S., Watson, M.S., Flockhart, D.A., Gage, B., et al. (2008) Pharmacogenetic Testing of CYP2C9 and VKORC1 Alleles for Warfarin. Genetics in Medicine, 10, 139-150. http://dx.doi.org/10.1097/GIM.0b013e318163c35f

\section{Abbreviations}

PPI: proton pump inhibitors

INR: international normalized ratio of prothrombin time 
Scientific Research Publishing (SCIRP) is one of the largest Open Access journal publishers. It is currently publishing more than 200 open access, online, peer-reviewed journals covering a wide range of academic disciplines. SCIRP serves the worldwide academic communities and contributes to the progress and application of science with its publication.

Other selected journals from SCIRP are listed as below. Submit your manuscript to us via either submit@scirp.org or Online Submission Portal.
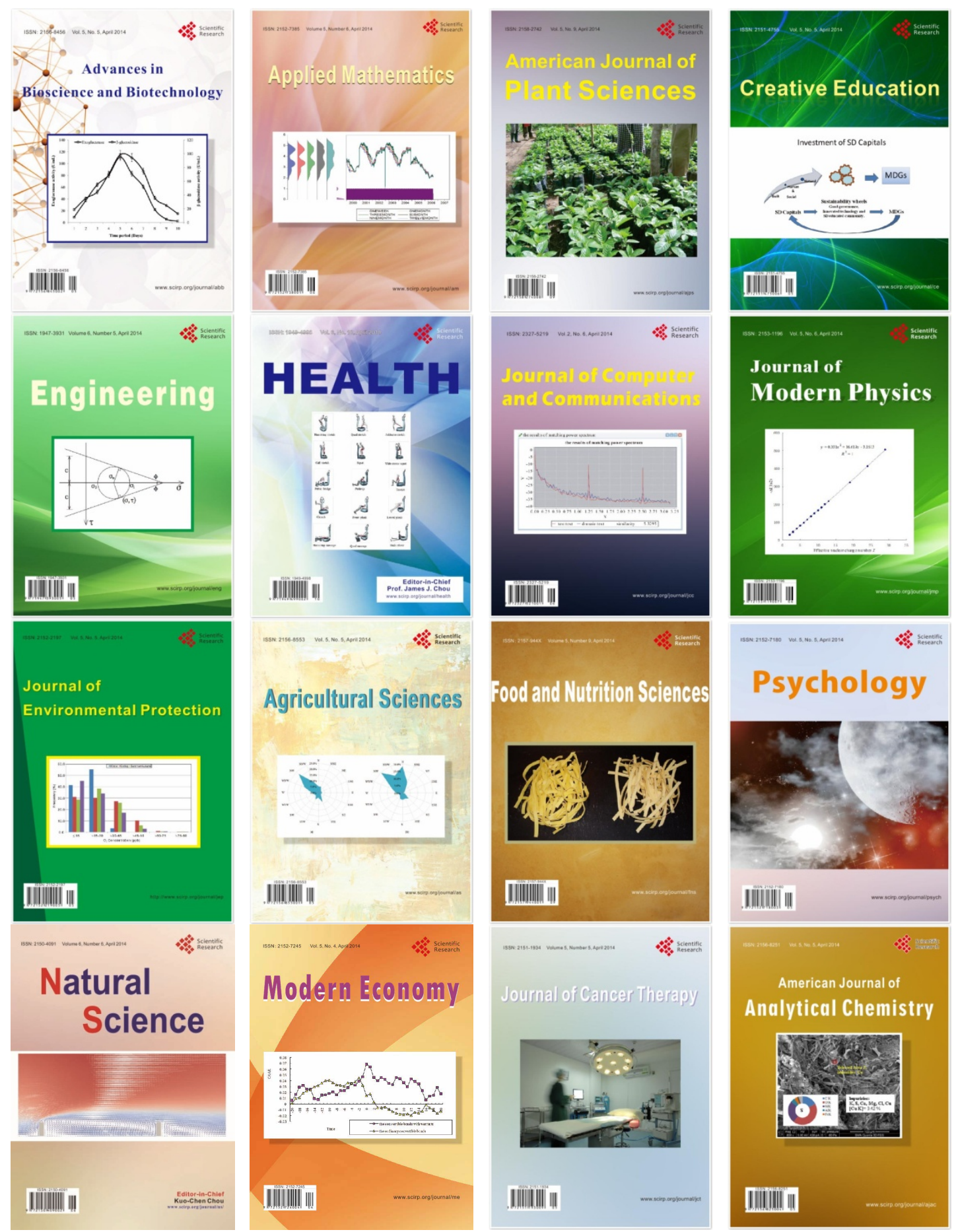